\title{
Vortex pattern and differential conductance in hole underdoped high-temperature superconductors with competing $d$-wave and antiferromagnetic orders
}

\author{
Lin Peng and Yongsheng Liu \\ School of Mathematics and Physics, Shanghai University of Electric Power, Shanghai 200090, China \\ E-mail: plpeng@shiep.edu.cn
}

Received March 26, 2013, revised April 30, 2013

\begin{abstract}
Vortex charges in copper oxide high-temperature superconductors with hole underdoping are investigated by Bogoliubov-de Gennes equations based on a model Hamiltonian with competing antiferromagnetic order and $d$-wave superconducting order (DSC) with variation of temperature. For the DSC, spin density wave (SDW) and charge density wave (CDW), the transition from stripe to checkerboard pattern and to 2D structure may occur with variation of temperature. Moreover, both spatial profile of the electron density distribution and intensity of the differential conductance are strongly affected by the SDW order around the vortex cores. The transitions from one-dimensional to isotropic two-dimensional structures of the vortex charge and spin-density wave orders may occur by tuning the temperature.

PACS: $74.78 . \mathrm{Bz}$ High- $T_{C}$ films;

74.72.Bk Y-based cuprates;

74.25.Qt Vortex lattices, flux pinning, flux creep.
\end{abstract}

Keywords: vortex charge, spin density wave, $d$-wave superconducting order.

\section{Introduction}

The vortex states in high-temperature superconductors (HTS) have received greater attention in recent years [1-9]. Since the parent compounds are antiferromagnetic (AFM) Mott insulators, novel physical properties of HTS including those in the vortex states would be expected due to the competition between more than one type of order parameter [10-20]. Theoretical formalism describing this effect is the Bogoliubov-de Gennes (BdG) approach, which can be viewed as real-space extension of the Bardeen-CooperSchrieffer (BCS) theory. This method allows one to reveal effects of imperfections in superconductors including impurities, surfaces and field-induced vortices. In the recent years there are numerous studies on the superconducting vortex lattice states using the discrete BdG equations and consequent diagonalization of the BdG mean-field Hamiltonian on a two-dimensional tight-binding lattice [5]. SDW orders with two-dimensional (2D) $[15,16]$ or stripe $[17,18]$ modulations are theoretically proposed to explain the observed checkerboard patterns. A negative vortex charge was predicted by Chen et al. for the slightly overdoped sample [11], which is consistent with the NMR measurements [10], but for the underdoped sample the theory is inconsistent with the NMR data: the experiment [10] indi- cates a positively charged core, whereas the theory [11] yields the "electron-rich" one. In a view of this discrepancy, Zhou et al. [19,20] investigated the effect of the longrange Coulomb repulsion on the AFM order and on the vortex charge. Their results show that a sign change from negative to positive might occur by tuning the long-range Coulomb repulsion strength or the doping parameter. Though above results may be relevant to some extent to the recent experimental observation, the influence of temperature on vortex charge was neglected and these results were obtained at zero temperature or at fixed temperature. Here we obtained experimental data for a certain range of temperature. In this work, we introduce the next-nearest-neighboring (NNN) hopping in the effective model Hamiltonian with competing $d$-wave superconducting (DSC) order and SDW orders, and investigate the effect of temperature on the AFM order and charged vortices for the holes underdoped HTS. Moreover, the differential conductance for HTS in confined geometry with the variance of temperature was also calculated. Our results show that the transition from strip structure to checkerboard pattern and the sign change in the vortex charge from negative to positive for underdoped case may occur with variation of temperature. 


\section{Methods}

We start with an effective model Hamiltonian in a twodimensional lattice, in which both the DSC and SDW orders are taken into account:

$$
\begin{gathered}
H=-\sum_{\langle i j\rangle, \sigma} t_{i j} c_{i \sigma}^{\dagger} c_{j \sigma}-\sum_{\langle\langle i j\rangle\rangle, \sigma} t_{i j}^{\prime} c_{i \sigma}^{\dagger} c_{j \sigma}+ \\
+\sum_{i, \sigma}\left[U\left\langle n_{i \sigma}\right\rangle-\mu\right] c_{i \sigma}^{\dagger} c_{j \sigma}+\sum_{\langle i j\rangle}\left(\Delta_{i j} c_{i \uparrow}^{\dagger} c_{j \downarrow}^{\dagger}+\Delta_{i j}^{*} c_{j \downarrow} c_{i \uparrow}\right),
\end{gathered}
$$

where the $t_{i j}$ and $t_{i j}^{\prime}$ are the nearest-neighboring $(\mathrm{NN})$ and NNN hopping integral, respectively, $c_{i \sigma}\left(c_{i \sigma}^{\dagger}\right)$ are destruction (creation) operators for electron of spin $\sigma, n_{i \sigma}=c_{i \sigma}^{\dagger} c_{i \sigma}$ is the number operator, and $\mu$ is the chemical potential determining the averaged electron density, $\bar{n}=\sum_{i, \sigma}\left\langle n_{i, \sigma}\right\rangle / N$ ( $N=N_{x} \times N_{y}$ is the linear dimension of the unite cell of the vortex lattice). $\langle i j\rangle$ denotes the summation over the NN sites and the site $j$ is the nearest neighbor to the site $i$, and the $\langle\langle i j\rangle\rangle$ denotes the summation over the NNN sites and the site $j$ is the next nearest neighbor to the site $i$, whereas the pairing attraction involves only the NN ones. The SDW and DSC orders have the following definitions, respectively: $\Delta_{i}^{S D W}=U\left\langle c_{i \uparrow}^{\dagger} c_{i \uparrow}-c_{i \downarrow}^{\dagger} c_{i \downarrow}\right\rangle, \quad \Delta_{i j}=V\left\langle c_{i \uparrow} c_{j \downarrow}-c_{i \downarrow} c_{j \uparrow}\right\rangle / 2$, where $U$ and $V$ represent, respectively, the on-site repulsion and the interaction strength for two orders. In the presence of magnetic field $B$, the hopping integral can be expressed as $t_{i j}=-t \exp \left(i \frac{\pi}{\Phi_{0}} \int_{\mathbf{r}_{j}}^{\mathbf{r}_{i}} A(\mathbf{r}) \cdot d \mathbf{r}\right)$. In the presence of a strong magnetic field, we assume the applied magnetic field to be uniform and choose a Landau gauge $\mathbf{A}=\frac{B}{2}(-y, x, 0)$. We restrict the NNN hopping integral to $\left|t_{i j}^{\prime}\right|<0.5\left|t_{i j}\right|$. Results for $\left|t_{i j}^{\prime}\right|>0.5\left|t_{i j}\right|$ would be unrealistic as the NNN element becomes comparable to the NN hopping. The meanfield Hamiltonian (1) can be diagonalized by solving the resulting BdG equations self-consistently:

$$
\sum_{j}^{N}\left(\begin{array}{cc}
H_{i j, \sigma} & \Delta_{i j} \\
\Delta_{i j}^{*} & -H_{i j, \bar{\sigma}}^{*}
\end{array}\right)\left(\begin{array}{c}
u_{j, \sigma}^{n} \\
v_{j, \bar{\sigma}}^{n}
\end{array}\right)=E_{n}\left(\begin{array}{c}
u_{i, \sigma}^{n} \\
v_{i, \bar{\sigma}}^{n}
\end{array}\right),
$$

where

$$
H_{i j, \sigma}=-t_{i j}-t_{i j}^{\prime}+\left[U_{i}\left\langle n_{i, \bar{\sigma}}\right\rangle-\mu\right] \delta_{i j}
$$

With the periodic boundary conditions [21] we can get the positive eigenvalues $\left(E_{n}\right)$ with eigenvectors $\left(u_{i \uparrow}^{n}, v_{i \downarrow}^{n}\right)$ and negative eigenvalues $\left(-E_{n}\right)$ with eigenvectors $\left(-v_{i \uparrow}^{n *}, u_{i \downarrow}^{n *}\right)$. The self-consistent conditions are

$$
\begin{gathered}
\left\langle n_{i \uparrow}\right\rangle=\sum_{n=1}^{2 N}\left|u_{i \uparrow}^{n}\right|^{2} f\left(\varepsilon_{n}\right), \\
\left\langle n_{i \downarrow}\right\rangle=\sum_{n=1}^{2 N}\left|v_{i \downarrow}^{n}\right|^{2}\left[1-f\left(\varepsilon_{n}\right)\right], \\
\Delta_{i j}=\sum_{n=1}^{2 N} \frac{V_{d}}{4}\left(u_{i \uparrow}^{n} v_{j \downarrow}^{n^{*}}+v_{i \downarrow}^{n^{*}} u_{j \uparrow}^{n}\right) \tanh \left(\frac{\varepsilon_{n}}{2 k_{B} T}\right),
\end{gathered}
$$

where $f(E)=1 /\left(\mathrm{e}^{E / k_{B} T}+1\right)$ is the Fermi distribution function. We then compute the DSC order parameter at the $i$-th site given by the expression

$$
\Delta_{i}^{D}=\frac{\Delta_{i+e_{x}, i}^{D}+\Delta_{i-e_{x}, i}^{D}-\Delta_{i, i+e_{y}}^{D}-\Delta_{i, i-e_{y}}^{D}}{4}
$$

where $\Delta_{i j}^{D}=\Delta_{i j} \exp \left(i \frac{\pi}{\Phi_{0}} \int_{\mathbf{r}_{i}}^{\left(\mathbf{r}_{i}+\mathbf{r}_{j}\right) / 2} A(\mathbf{r}) \cdot d \mathbf{r}\right)$ and $e_{x, y}$ the unit vector along the $(x, y)$ direction. In order to be able to discuss the results of STM experiments performed in the presence of vortex lines, we need a model of the tunneling current between the microscope tip and the surface of the sample. This current is related to the one-particle spectral functions in the tip of the microscope and in the superconductor. The latter can be expressed in terms of the quasiparticle amplitudes [22]:

$$
A_{S}(i, E)=2 \pi \sum_{n}\left[\left|u_{i, \uparrow}^{n}\right|^{2} \delta\left(E-E_{n}\right)+\left|v_{i, \downarrow}^{n}\right|^{2} \delta\left(E-E_{n}\right)\right]
$$

We assume here that the presence of the surface does not affect the quasi-particle amplitudes of the superconductor, so we can use the solutions of Eq. (2). We also assume the spectral function of the tip to be that of a simple metal:

$$
A_{N}(i, E)=2 \pi \sum_{k} \delta\left(E-E_{k}\right)
$$

If the tip is small compared to flux core dimensions, and is centered at position $\mathbf{r}$, the tunneling current due to an applied voltage $V$ is

$$
I(i, V) \propto \int \frac{d E}{2 \pi} A_{S}(i, E) A_{N}(i, E+e V) .
$$

Using the Bogoliubov transformation, its derivative is expressed in terms of the $u$ and $v$ amplitudes:

$$
\frac{\partial I(i, V)}{\partial V} \propto-\sum_{n}\left[\left|u_{i, \uparrow}^{n}\right|^{2} \frac{1}{\cosh ^{2} \frac{E_{n}-e V}{2 k_{B} T}}-\left|v_{i, \downarrow}^{n}\right|^{2} \frac{1}{\cosh ^{2} \frac{E_{n}+e V}{2 k_{B} T}}\right] .
$$


In our calculation, the average electron density is fixed at $\bar{n} \approx 0.873$ by adjusting the chemical potential, i.e., the hole doping $\delta=1-\bar{n}=0.127$. The distance is measured in units of the lattice constant $a$, and we take $k_{B}=a=t=1$ for simplicity. In order to emphasize the significance of AFM competing with DSC order, we choose a large $U(=2.4)$ and a intermediate $V(=1)$. The NNN hopping integral parameter $\left|t_{i j}^{\prime}\right|=0.2\left|t_{i j}\right|$, which has been chosen to fit the band structure of most cuprate superconductors. The linear dimension of the unit cell of the vortex lattice is $N_{x} \times N_{y}=48 \times 24$. This choice corresponds to the magnetic field $B \simeq 24 \mathrm{~T}$. The amplitude of the magnetic field is determined by the condition that each magnetic unit cell contains two superconducting flux quanta, i.e., $B=2 \Phi_{0} /\left(N_{x} N_{y} a^{2}\right)$. The main procedure of self consistency calculation is summarized as follows. For a given initial set of parameters $n_{i \sigma}$ and $\Delta_{i j}$, the Hamiltonian is numerically diagonalized and the electron wave functions obtained are used to calculate the new parameters for the next iteration step. The calculation is repeated until the relative difference of order parameter between two consecutive iteration steps is less than $10^{-4}$.

\section{Results and discussions}

Figure 1 plots the spatial profiles of the superconductivity order parameter [(a), (d), (g) and (j)], staggered magnetization [(b), (e), (h) and (k)], and the relative charge density [(c), (f), (i) and (l)] near the vortex cores in a 48×24 lattice. Here, the staggered magnetization of the induced AFM or SDW order is defined as $M_{i}^{S}=(-1)^{i}\left\langle n_{i, \uparrow}-n_{i, \downarrow}\right\rangle$. The vortex centers are situated at site $(12,12)$ and $(12,24)$, respectively. Around the core centers the AFM orders are generated and the DSC orders are partially suppressed. The first panels [Figs. 1 (a) - (c)] are for $T=0.025$, the second panels [Figs. 1(d)-(f)] are for $T=0.05$, the third panels [Figs. 1(g)-(i)] are for $T=0.075$, and the fourth panels
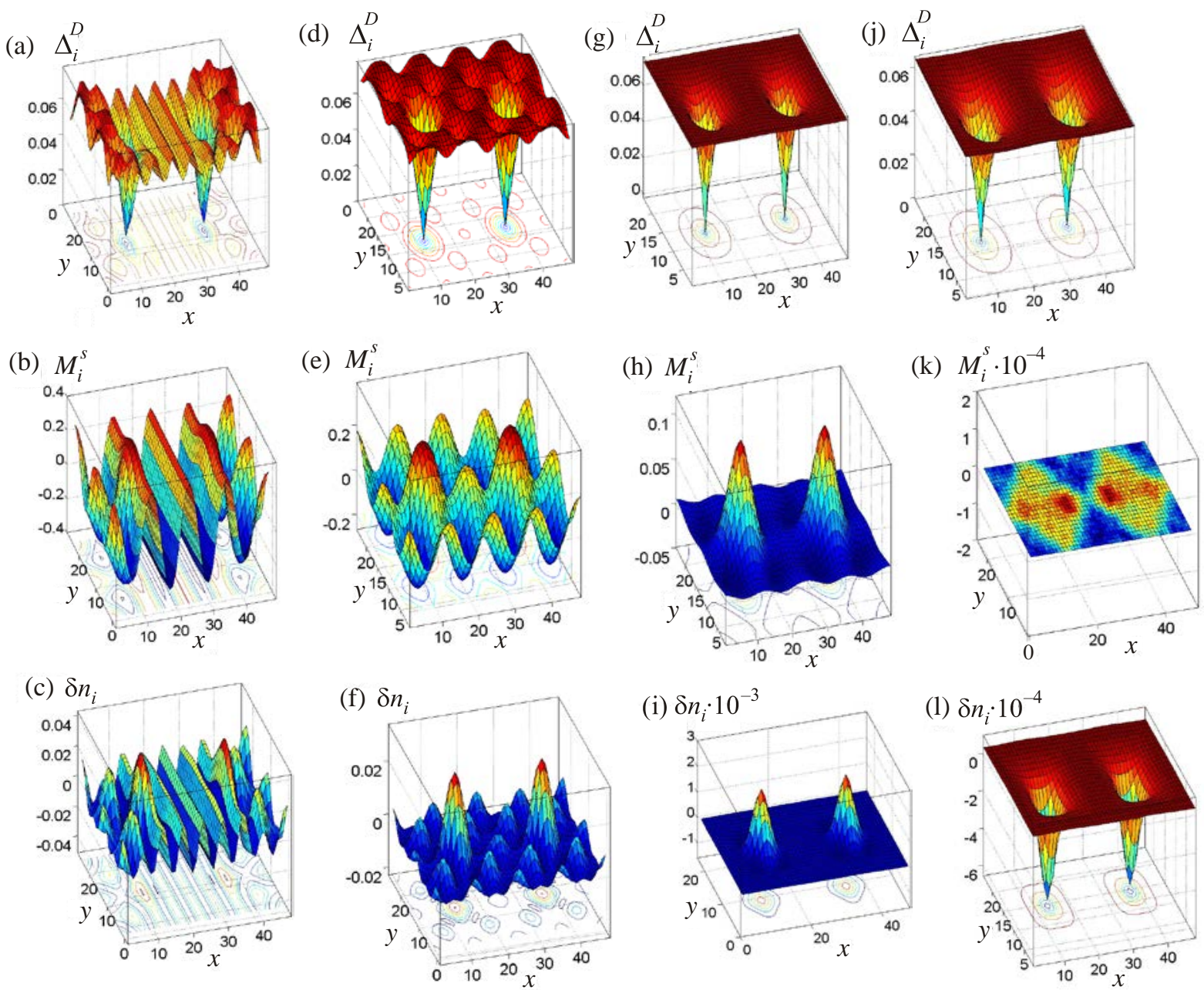

Fig. 1. (Color online) Spatial variations of the DSC order parameter [(a), (d), (g) and (j)], staggered magnetization [(b), (e), (h) and (k)], and the charge density [(c), (f), (i) and (l)] near the vortex core in a 48×24 lattice. The first panels [(a)-(c)], second panels [(d)-(f)], third panels [(g)-(i)], and fourth panels [(j)-(l)] are for $T=0.025,0.05,0.075$, and $0.1 \mathrm{~K}$, respectively. The other parameter values are $\bar{n}=$ $=0.873, U=2.4, V=1, t^{\prime}=-0.2$. 
[Figs. 1(j)-(l)] are for $T=0.1$. As can be seen at the first panels of Figs. 1(a)-(c) $(T=0.025)$, the DSC orders show a $y$-axis-oriented stripe-like structure. We notice that the striped SDW order oscillates with a period of $12 a$ [see Fig. 1(a)], while the charge orders exhibit an oscillation with a period of 6a [see Fig. 1(c)], half of that of the SDW, along the $x$ direction. The results are in qualitative agreement with the observations [23]. We should mention that the periods of SDW and charge orders obtained from the experiments $[13,24]$ are $8 a$ and $4 a$ for an optimally doped sample, which could be reproducible by tuning $U$ and the NNN hopping strength [5]. With further increasing of the $T(T=0.05)$, we found a transition from stripe-like structure to checkerboard-like structure. The results are in qualitative agreement with the experimental [25] and theoretical [13] results, as shown in the second panels of Figs. 1(d)-(f). At the third panels of Figs. 1(g)-(i) $(T=0.075)$, the checkerboard-like structures vanish and the isotropic two-dimensional behaviors are formed. These results indicate that the transition from the stripe structure to the checkerboard pattern and the isotropic two-dimensional behavior for the spatial profile of the electron density distribution, could be predicted when the SDW order around the vortex cores decrease. The fourth panels in Figs. 1(j)-(l) are for $T=0.1$, where the AFM orders are extremely weak $\left(\left|M_{i}^{S}\right|<10^{-3}\right)$ and the vortex charges are positive (hole-like). This indicates that the AFM order is decreased remarkably around the vortex cores, expelling the electrons from the vortex cores into the bulk of the superconductor and the vortex charge become positive. In this case, HTS may be regarded as a system with two types of electronic subsystems. One type is superconducting and the other with the core size in the order of coherence length remains normal. The chemical-potential differences are present between these subsystems, which drive the electrons outside from the core, resulting in positively charged cores. Hence, the vortex core will be negatively charged for hole underdoped HTS at the lower temperature $T$ where the AFM and DSC orders coexist [26], whereas positive core charges are expected for relatively high temperature where AFM order is vanishing and larger ferromagnetic (FM) islands accommodate the holes [27]. So we believe that the experimental results for the underdoped sample [10] where positively charged vortex cores are reported may be understood based on our calculation when the appropriate $T$ and the NNN hoping strength are included. In addition, compared to hole overdoped sample [28], the positive charge decrease one order of magnitude for underdoped sample. This could be attributed to relatively large SDW order with the increase of electron concentration [29], which reduces the number of holes in the vortex cores.

We plotted the differential conductance according to Eq. (12) in Fig. 2. Figure 2 shows the energy dependence of the differential conductance $\partial I / \partial V$ at the vortex core center $(12,12)$ and the mid-point $(12,24)$ between two nearest-neighbor vortices along the $x$ direction for the va-
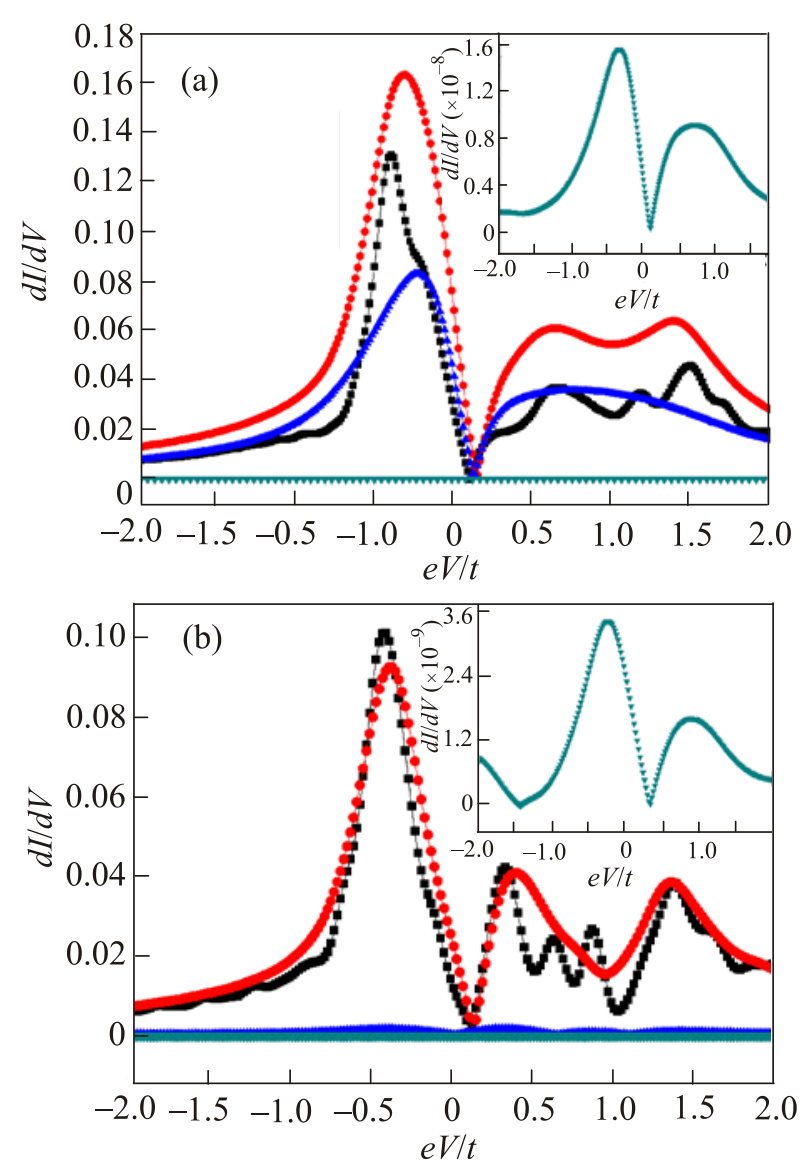

Fig. 2. (Color online) The differential conductance as a function of energy with various $T$, K: $0.025(\boldsymbol{\bullet}), 0.05(\bullet), 0.075(\boldsymbol{\Delta})$, $0.10(\boldsymbol{\nabla})$; (a) - at the core center $(12,12)$ and (b) - at the midpoint $(12,24)$. Inset: region expanded at $T=0.10$.

riance of $T$. As can be seen from Fig. 2(a), the presence of the AFM order provides a mechanism for splitting of the zero-bias conductance peak into local peaks at the core center $(12,12)$ and the mid-point $(12,24)$. We note that the intensity of the peak located at the negative energy is much larger than that of the peak located at the positive energy at the vortex core center $(12,12)$, and the same is true for the mid-point $(12,24)$ (see Fig. 2(b)). The intensity of differential conductance peaks is strongly affected by the AFM order around vortex cores. The AFM order around vortex cores decreases and vanish rapidly when the temperature increases and the larger ferromagnetic islands are forming [27], leading to increase of the resistance (see the inset in Fig. 2).

\section{Conclusion}

In conclusion, we use BdG equations to investigate vortex charges in hole underdoped HTS with variation of temperature $T$. The spatial variations of the DSC, the SDW and the CDW indicate the transition from stripe to checkerboard pattern and to 2D structure with increase of temperature. The vortex cores will be negatively charged for hole 
underdoped HTS at the lower temperature $T$ where the AFM and DSC orders coexist, whereas positive core charges are expected for relatively higher temperature where AFM order vanishes. The SDW orders around vortex cores decrease rapidly when the temperature increases, while the intensity of differential conductance declines and vanishes with increase of $T$. These results could provide some good understanding for the novel phenomena in experimental observation.

\section{Acknowledgments}

This work is supported by the National Natural Science Foundations of Shanghai (No. 13ZR1417600) and the Startup Fund for Talented Scholars of Shanghai University of Electric Power (No. K2011-014), and the Shanghai Science Fund for the Excellent Young Teachers (No. Z2012-012).

1. J.S. Jiang, D. Davidović, H. Reich Daniel, and C.L. Chien, Phys. Rev. Lett. 74, 314 (1995).

2. T. Kontos, M. Aprili, J. Lesueur, and X. Grison, Phys. Rev. Lett. 86, 304 (2001).

3. Z.C. Dong, D.Y. Xing, and J. Dong, Phys. Rev. B 65, 214512 (2002).

4. L. Peng, C. Cai, C. Chen, Z. Liu, and B. Gao, Phys. Lett. A 373, 2273 (2009).

5. G. Zha, Y. Chen, F.M. Peeters, and S. Zhou, Phys. Rev. B 80, 064518 (2009).

6. L. Peng, Y. Liu, C. Cai, and J. Zhang, Chin. Phys. Lett. 28, 087401 (2011).

7. L. Peng, Y. Liu, T. Gao, C. Cai, and J. Zhang, J. Supercond. Nov. Magn. 24, 1683 (2011).

8. L. Peng and Y. Liu, Appl. Phys. A 104, 1255 (2011).

9. L.R. Tagirov, Phys. Rev. Lett. 83, 2058 (1999).

10. K.I. Kumagai, K. Nozaki, and Y. Matsuda, Phys. Rev. B. 63, 144502 (2001).

11. Y. Chen, Z.D. Wang, J.X. Zhu, and C.S. Ting, Phys. Rev. Lett. 89, 217001 (2002).
12. J.M. Tranquada, B.J. Sternlleb, J.D. Axe, Y. Nakamura, and S. Uchida, Nature 375, 561 (1995).

13. B. Lake, G. Aeppli, K.N. Clausen, D.F. McMorrow, K. Lefmann, N.E. Hussey, M. Nohara, H. Takagi, T.E. Mason, and A. Schröder, Science 291, 1759 (2001).

14. K. McElroy, D.-H. Lee, J.E. Hoffman, K.M. Lang, J. Lee, E.W. Hudson, H. Eisaki, S. Uchida, and J.C. Davis, Phys. Rev. Lett. 94, 197005 (2005).

15. H.D. Chen, J.P. Hu, S. Capponi, E. Arrigoni, and S.C. Zhang, Phys. Rev. Lett. 89, 137004 (2002).

16. A. Polkovnikov, M. Vojto, and S. Sachdev, Phys. Rev. B 65, 220509 (2002).

17. Y. Chen, H.Y. Chen, and C.S. Ting, Phys. Rev. B 66, 104501 (2002).

18. D. Podolsky, E. Demler, K. Damle, and B.I. Halperin, Phys. Rev. B 67, 094514 (2002).

19. H. Zhao, G. Zha, S. Zhou, and F.M. Peeters, Phys. Rev. B 78, 064505 (2008).

20. G. Zha, H. Zhao, and S. Zhou, Phys. Rev. B 76, 132503 (2007).

21. S. Zhou, Chin. Phys. 10, 541 (2001).

22. F. Gygi and M. Schlüter, Phys. Rev. B 43, 7609 (1991).

23. B. Lake, H.M. Ronnow, N.B. Christensen, G. Aeppli, K. Lefmann, D.F. McMorrow, P. Vorderwisch, P. Smeibidl, N. Mangkorntong, T. Sasagawa, M. Nohara, H. Takagi, and T.E. Mason, Nature (London) 415, 299 (2002).

24. J.E. Hoffman, E.W. Hudson, K.M. Lang, V. Madhavan, S.H. Pan, H. Eisaki, S. Uchida, and J.C. Davis, Science 295, 466 (2002).

25. D.P. Arovas, A.J. Berlinsky, C. Kallin, and S.C. Zhang, Phys. Rev. Lett. 79, 2871 (1997).

26. V.F. Mitrovic, E.E. Sigmund, M. Eschrig, H.N. Bachman, W.P. Halperin, A.P. Reyes, P. Kuhns, and W.G. Moulton, Nature (London) 413, 505 (2001).

27. M. Civelli, M. Capone, S.S. Kancharla, O. Parcollet, and G. Kotliar, Phys. Rev. Lett. 95, 106402 (2005).

28. L. Peng, Y. Liu, C. Chen, H. Li, C. Jia, and Q. Zou, J. Low Temp. Phys. 170, 91 (2013).

29. L. Peng, Y. Liu, H. Hu, Y. Zhang, Y. Xu, and Z. Liu, J. Supercond. Nov. Magn. 26, 321 (2013). 